ITEP-TH-21/13

FIAN/TD-11/13

\title{
Three-particle Integrable Systems with Elliptic Dependence on Momenta and Theta Function Identities
}

\author{
G. Aminov ${ }^{*} \quad$ A. Mironov ${ }^{\dagger} \quad$ A. Morozov ${ }^{\ddagger} \quad$ A. Zotov ${ }^{\S}$
}

\begin{abstract}
We claim that some non-trivial theta-function identities at higher genus can stand behind the Poisson commutativity of the Hamiltonians of elliptic integrable systems, which were introduced in 1, 2] and are made from the theta-functions on Jacobians of the Seiberg-Witten curves. For the case of three-particle systems the genus- 2 identities are found and presented in the paper. The connection with the Macdonald identities is established. The genus-2 theta-function identities provide the direct way to construct the Poisson structure in terms of the coordinates on the Jacobian of the spectral curve and the elements of its period matrix. The Lax representations for the two-particle systems are also obtained.
\end{abstract}

\section{Introduction}

We suggest a new approach to obtain the multi-particle generalization of p,q-dual integrable systems constructed in [1, 3]. The list of these systems includes duals to elliptic Calogero [4] and elliptic Ruijsenaars models [5]. The most interesting are the double-elliptic integrable systems [1, 2, 6, 17, where both coordinates and momenta take values on elliptic curves. From the point of view of the low-energy effective actions of Yang-Mills theories [8, 9] the integrable systems under consideration are associated with (compactified) six-dimensional SUSY gauge theories [10, 11, 12, 13, 14, 15, 16, 17, 18, and are strongly involved in modern discussion of the Seiberg-Witten theory [19, 20, 21, 22, 23, 24, 25, 26, 27].

The main claim of our paper is the existence of some new theta-function identities which stand behind the Poisson commutativity of the Hamiltonians considered in [1, 2]. We present such relations for the case

*Institute of Theoretical and Experimental Physics, Moscow, Russia and Moscow Institute of Physics and Technology, Dolgoprudny, Russia. E-mail: aminov@itep.ru

$\dagger$ Theory Department, Lebedev Physics Institute and Institute of Theoretical and Experimental Physics, Moscow, Russia. E-mail: mironov@itep.ru,mironov@lpi.ac.ru

${ }^{\ddagger}$ Institute of Theoretical and Experimental Physics, Moscow, Russia. E-mail: morozov@itep.ru

$\S$ Institute of Theoretical and Experimental Physics and Steklov Mathematical Institute, RAS, Moscow, Russia; Moscow Institute of Physics and Technology, Dolgoprudny, Russia. E-mail: zotov@itep.ru 
of three-particle systems. Unfortunately, it is not clear, how to prove them even in this situation, but these relations seem to be rather interesting by themselves - they can be considered as a genus two generalization of the Macdonald identities [28]. Also, these theta-function identities provide a direct way to construct the Poisson structure in terms of the coordinates on the Jacobian of the spectral curve and the elements of its period matrix. In the case of three-particle systems we reduce the problem of constructing the Poisson brackets to the problem of solving the system of three partial differential equations with respect to the pair of unknown functions.

In section 2 we discuss the Hamiltonians introduced in [1, 2]. The whole construction is restricted to the Sieberg-Witten families of the spectral curves and the Poisson commutativity of the Hamiltonians is related to the Sieberg-Witten prepotential. In [2] this commutativity was checked in the first non-trivial example in the first several orders of $\Lambda / a$-expansion - and this check was relied severely on the known shape of the Seiberg-Witten prepotential. In section 3 we propose another approach, that deals with arbitrary Riemann surfaces instead of the Sieberg-Witten curves. Then the Poisson commutativity of the Hamiltonians is due to some new theta-function identities, which seem to be true for an arbitrary Riemann surface. In sections 4 and 5 we present the theta-function identities of genus two for the case of three-particle systems and reveal some interesting mathematical structures behind them. In section 6 the connection with the Ruijsenaars models is established by means of some specific trigonometric limits. In section 7 we go back to the case of two-particle systems and construct the Lax representation using the original Ruijsenaars idea [29].

\section{Hamiltonians and Seiberg-Witten data}

The Hamiltonians of the systems dual to the elliptic Calogero and Ruijsenaars models with an elliptic dependence on momenta as well as the self-dual double elliptic systems were introduced in [1, 2]. In the case of $N=3$ particles in the center of a mass frame one has two Hamiltonians of the form

$$
H_{1}(\mathbf{z} \mid \Omega)=\frac{\theta_{11}^{(2)}(\mathbf{z} \mid \Omega)}{\theta^{(2)}(\mathbf{z} \mid \Omega)}, \quad H_{2}(\mathbf{z} \mid \Omega)=\frac{\theta_{22}^{(2)}(\mathbf{z} \mid \Omega)}{\theta^{(2)}(\mathbf{z} \mid \Omega)},
$$

where we use the following notations for the Riemann theta function with symmetric $2 \times 2$ period matrix $\Omega$ :

$$
\begin{gathered}
\theta^{(2)}(\mathbf{z} \mid \Omega)=\sum_{\mathbf{n} \in \mathbb{Z}^{2}} \mathrm{e}\left(\frac{1}{2} \mathbf{n}^{t} \Omega \mathbf{n}+\mathbf{n} \cdot \mathbf{z}\right), \quad \mathbf{z}^{t}=\left(z_{1}, z_{2}\right), \quad \mathrm{e}(x) \equiv \exp (2 \pi \imath x), \\
\theta_{\mathbf{c}}^{(2)} \equiv \theta^{(2)}\left[\begin{array}{c}
\mathbf{0} \\
\mathbf{c} / 3
\end{array}\right]=\sum_{\mathbf{n} \in \mathbb{Z}^{2}} \mathrm{e}\left(\frac{1}{2} \mathbf{n}^{t} \Omega \mathbf{n}+\mathbf{n} \cdot\left(\mathbf{z}+\frac{\mathbf{c}}{3}\right)\right), \quad \mathbf{c} \in \mathbb{Z}_{3}^{2} .
\end{gathered}
$$

The role of the non-commutative coordinates and momenta in these Hamiltonians is played by the elements of the period matrix $\Omega$ and the coordinates on the Jacobian $\mathbf{z}$. The hypothesis of [1] is that the Hamiltonians are Poisson commuting with respect to the Seiberg-Witten symplectic structure

$$
\omega^{S W}=\sum_{i=1}^{N} \mathrm{~d} \hat{p}_{i} \wedge \mathrm{d} a_{i}
$$

where $\hat{p}_{i}=z_{i}+\frac{1}{N} \sum_{j=1}^{N} \hat{p}_{j}, z_{3} \equiv-z_{1}-z_{2}$ and $\sum_{i=1}^{N} a_{i}=0$, while the $N(N-1) / 2=3$ elements of the period matrix $\Omega$ are not arbitrary, but functions of just $N-1=2$ flat moduli:

$$
\Omega=\Omega(\mathbf{a})
$$


Following this idea one can assume the Poisson brackets in terms of the coordinates $\left(z_{1}, z_{2}\right)$ and the elements $\Omega_{i j}$ to be of the form

$$
\left\{z_{i}, \Omega_{j k}\right\}=P_{i j k}, \quad\left\{z_{i}, z_{j}\right\}=0, \quad\left\{\Omega_{i j}, \Omega_{k l}\right\}=0 .
$$

Notice that the elements $P_{i j k}$ are again functions of the flat moduli only. Thus, we can consider them to be the functions of the period matrix $\Omega$ as well:

$$
P_{i j k}=P_{i j k}(\Omega) \text {. }
$$

Taking into account the connection of the period matrix $\Omega$ with the Seiberg-Witten prepotential

$$
\Omega_{i j}=\frac{\partial^{2} \mathcal{F}}{\partial a_{i} \partial a_{j}}, \quad i, j \in\{1,2\}
$$

and the assumption [1] that the prepotential is Poisson-commuting with the total momentum of the system, we get the following relation:

$$
P_{i j k}=\frac{\partial^{3} \mathcal{F}}{\partial a_{i} \partial a_{j} \partial a_{k}}, \quad i, j, k \in\{1,2\} .
$$

In the Seiberg-Witten theory these third derivatives are usually given by the residue formulas and can be expressed through theta-constants, see, for example, [30].

\section{Poisson brackets}

In this section we forget about the Seiberg-Witten prepotential and investigate, what can be achieved with the help of (2.6) and (2.7) only. So, we consider $\Omega$ as an arbitrary period matrix of genus-2 Riemann surface.

The elements of the Poisson structure (2.6) $P_{i j k}$ are unknown. To derive them as functions of $\Omega$, we use two necessary conditions:

1. the Poisson commutativity of Hamiltonians (2.1),

2. the Jacobi identity.

To impose the first condition we formulate the Poisson bracket between the Hamiltonians (2.1) in terms of the coordinates $\mathbf{z}$ and the elements of the period matrix:

$$
\left\{H_{1}, H_{2}\right\}=\sum_{i=1}^{2} \sum_{j \leqslant k} P_{i j k}\left(\frac{\partial H_{1}}{\partial z_{i}} \frac{\partial H_{2}}{\partial \Omega_{j k}}-\frac{\partial H_{2}}{\partial z_{i}} \frac{\partial H_{1}}{\partial \Omega_{j k}}\right) .
$$

Then the strategy of finding $P_{i j k}$ is the following. The Poisson commutativity of the Hamiltonians $\left\{H_{1}, H_{2}\right\}=$ 0 means that there are some relations on genus two theta functions with coefficients depending only on the elements of the period matrix. Hence, to satisfy the first condition we search for the following relations on genus two theta functions:

$$
\sum_{i=1}^{2} \sum_{j \leqslant k} P_{i j k}^{\alpha}\left(\frac{\partial H_{1}}{\partial z_{i}} \frac{\partial H_{2}}{\partial \Omega_{j k}}-\frac{\partial H_{2}}{\partial z_{i}} \frac{\partial H_{1}}{\partial \Omega_{j k}}\right)=0, \forall \mathbf{z}, \Omega
$$


where index $\alpha$ enumerates the relations and elements $P_{i j k}^{\alpha}$ are some new functions of $\Omega$ which can be used for constructing $P_{i j k}$ in the form

$$
\left\{z_{i}, \Omega_{j k}\right\}=P_{i j k}=\sum_{\alpha} F_{\alpha} P_{i j k}^{\alpha}, F_{\alpha}=F_{\alpha}(\Omega) .
$$

As we mentioned earlier, it is natural to assume that the coefficients $P_{i j k}^{\alpha}$ are some theta-constants. Thus, the relations (3.2) are made out of the theta functions, theta-constants and their derivatives. Also, the left hand side of (3.2) should vanish for arbitrary values of $\mathbf{z}$ and $\Omega$, implying that our relations are actually some theta-function identities. It is natural to wonder, if any identities of such form exist without a direct reference to the Seiberg-Witten theory.

Indeed, we found two relations on genus-2 theta functions (2.1) and genus-2 theta constants $P_{i j k}^{\alpha}, \alpha=1,2$, which satisfy all the symmetry conditions arising from the previous section. The relations are linearly independent, but the second relation can be obtained from the first one by permutating the diagonal elements of the matrix $\Omega$ and the coordinates $\left(z_{1}, z_{2}\right)$. Unfortunately, it is not clear for us how to prove these relations in general. But even the first simple analysis shows some interesting mathematical structures behind them. We will present some details on these structures in the next two sections.

Once the relations on genus-2 theta functions with coefficients $P_{i j k}^{\alpha}$ are found, we impose the second condition (the Jacobi identity) in order to find $F_{\alpha}(\Omega)$ (3.3). It takes the form

$$
\sum_{m \leqslant n}\left(F_{1} P_{1 m n}^{1}+F_{2} P_{1 m n}^{2}\right) \partial_{\Omega_{m n}}\left(F_{1} P_{2 i j}^{1}+F_{2} P_{2 i j}^{2}\right)=\sum_{m \leqslant n}\left(F_{1} P_{2 m n}^{1}+F_{2} P_{2 m n}^{2}\right) \partial_{\Omega_{m n}}\left(F_{1} P_{1 i j}^{1}+F_{2} P_{1 i j}^{2}\right),
$$

where $i \leqslant j, i, j \in\{1,2\}$. Thus, we reduce the problem of constructing the Poisson brackets to the problem of solving the system of three partial differential equations with respect to the unknown functions $F_{\alpha}, \alpha=1,2$. Of course, it is a challenging issue to find the set of all solutions of the system (3.4) and to understand whether the systems under consideration are unique or not. We leave this problem for future investigation.

\section{Theta-function identities of genus 2}

In this section we discuss the identities (3.2) on the genus two Riemann theta functions and present the elements $P_{i j k}^{\alpha}, \alpha=1,2$ as a solution of the system of linear equations. To describe the first relation from (3.2) with coefficients $P_{i j k}^{1}$ we use the symmetry conditions generated by (2.9)

$$
P_{112}^{1}=P_{211}^{1}=P_{121}^{1}, \quad P_{212}^{1}=P_{122}^{1}=P_{221}^{1}
$$

and additionally assume that

$$
P_{222}^{1}=0 .
$$

These conditions leaves us with 3 independent unknown components of $P^{1}: P_{111}^{1}, P_{112}^{1}$ and $P_{212}^{1}$. Then substituting some particular values of $\mathbf{z}$ in (3.2), we get the system of linear equations on elements $P_{i j k}^{1}$. Therefore, it is enough to consider two different values of $\mathbf{z}$ :

$$
\mathbf{z}_{1}=(0,0), \quad \mathbf{z}_{2}=\left(0, \frac{1}{3}\right)
$$

which will give the expressions of $P_{i j k}^{1}$ in terms of the derivatives of the following functions at the point $(0,0)$ :

$$
H=H_{1}=\frac{\theta_{11}^{(2)}(\mathbf{z} \mid \Omega)}{\theta^{(2)}(\mathbf{z} \mid \Omega)}=\frac{\theta_{22}^{(2)}(-\mathbf{z} \mid \Omega)}{\theta^{(2)}(-\mathbf{z} \mid \Omega)}, \quad F=H_{1}\left(\mathbf{z}+\mathbf{z}_{2}\right)=\frac{\theta_{12}^{(2)}(\mathbf{z} \mid \Omega)}{\theta_{01}^{(2)}(\mathbf{z} \mid \Omega)}, \quad G=H_{2}\left(\mathbf{z}+\mathbf{z}_{2}\right)=\frac{\theta_{20}^{(2)}(\mathbf{z} \mid \Omega)}{\theta_{01}^{(2)}(\mathbf{z} \mid \Omega)} .
$$


Solution of the above-described system of two linear equations with fixed arbitrary factor can be presented in the following way:

$$
P_{111}^{1}=a_{4} b_{3}-a_{3} b_{4}, \quad P_{112}^{1}=P_{211}^{1}=a_{3} b_{1}-a_{1} b_{3}, \quad P_{212}^{1}=P_{122}^{1}=a_{1} b_{4}-a_{4} b_{1}, \quad P_{222}^{1}=0,
$$

where

$$
\begin{gathered}
a_{1}=\left.\left(\frac{\partial H}{\partial z_{1}} \frac{\partial H}{\partial \Omega_{11}}\right)\right|_{\mathbf{z}=0}, \quad a_{3}=\left.\left(\frac{\partial H}{\partial z_{1}} \frac{\partial H}{\partial \Omega_{22}}+\frac{\partial H}{\partial z_{2}} \frac{\partial H}{\partial \Omega_{12}}\right)\right|_{\mathbf{z}=0}, \quad a_{4}=\left.\left(\frac{\partial H}{\partial z_{2}} \frac{\partial H}{\partial \Omega_{11}}+\frac{\partial H}{\partial z_{1}} \frac{\partial H}{\partial \Omega_{12}}\right)\right|_{\mathbf{z}=0}, \\
b_{1}=\left.\left(\frac{\partial F}{\partial z_{1}} \frac{\partial G}{\partial \Omega_{11}}-\frac{\partial G}{\partial z_{1}} \frac{\partial F}{\partial \Omega_{11}}\right)\right|_{\mathbf{z}=0}, \quad b_{3}=\left.\left(\frac{\partial F}{\partial z_{1}} \frac{\partial G}{\partial \Omega_{22}}-\frac{\partial G}{\partial z_{1}} \frac{\partial F}{\partial \Omega_{22}}+\frac{\partial F}{\partial z_{2}} \frac{\partial G}{\partial \Omega_{12}}-\frac{\partial G}{\partial z_{2}} \frac{\partial F}{\partial \Omega_{12}}\right)\right|_{\mathbf{z}=0} \\
b_{4}=\left.\left(\frac{\partial F}{\partial z_{2}} \frac{\partial G}{\partial \Omega_{11}}-\frac{\partial G}{\partial z_{2}} \frac{\partial F}{\partial \Omega_{11}}+\frac{\partial F}{\partial z_{1}} \frac{\partial G}{\partial \Omega_{12}}-\frac{\partial G}{\partial z_{1}} \frac{\partial F}{\partial \Omega_{12}}\right)\right|_{\mathbf{z}=0} .
\end{gathered}
$$

The second relation with coefficients $P_{i j k}^{2}$ can be obtained from the first one by permutating the diagonal elements of matrix $\Omega$ and the coordinates $\left(z_{1}, z_{2}\right)$. Taking into account that the Hamiltonians (2.1) are invariant under this permutation, we get the following answer for the elements $P_{i j k}^{2}$ :

$$
P_{222}^{2}=a_{3} b_{4}-a_{4} b_{3}, \quad P_{112}^{2}=P_{211}^{2}=a_{2} b_{3}-a_{3} b_{2}, \quad P_{212}^{2}=P_{122}^{2}=a_{4} b_{2}-a_{2} b_{4}, \quad P_{111}^{2}=0,
$$

where

$$
a_{2}=\left.\left(\frac{\partial H}{\partial z_{2}} \frac{\partial H}{\partial \Omega_{22}}\right)\right|_{\mathbf{z}=0}, \quad b_{2}=\left.\left(\frac{\partial F}{\partial z_{2}} \frac{\partial G}{\partial \Omega_{22}}-\frac{\partial G}{\partial z_{2}} \frac{\partial F}{\partial \Omega_{22}}\right)\right|_{\mathbf{z}=0} .
$$

Finally, we obtain the identities (3.2) with the coefficients $P_{i j k}^{\alpha}$ (4.5)-(4.10). We emphasize that all the elements in the formula (3.2) are expressed through the genus-2 theta functions, therefore (3.2) is actually a theta-function identity (we do not present it explicitly just to save the space - it is somewhat lengthy). Despite $p-q$ duality requires it to be true only for the special 2-parametric family of period matrices $\Omega(\mathbf{a})$, we claim that it holds on entire 3-dimensional space of period matrices $\Omega$ and for arbitrary z. To confirm this statement we checked it in 14 orders of expansion in $Q_{1} \equiv \mathrm{e}\left(\Omega_{11} / 2\right)$ and $Q_{2} \equiv \mathrm{e}\left(\Omega_{22} / 2\right)$, what leaves us little doubt that they are correct. However, a general proof is still an open problem.

As an additional evidence in support of these identities we consider their reduction to the genus-one theta-constants - one would naturally expect some trivial well-known identity to occur. What happens - it is indeed a known, but rather advanced and interesting Macdonald identity from [28].

\section{The Macdonald identities}

It turns out that the relations (3.2) can be considered as the genus two generalizations of the Macdonald identities [28]. To show this connection we go backwards: degenerate the initial spectral curve into two different elliptic curves by taking the limit $\Omega_{12} \rightarrow 0$. Then consider the trigonometric limit $\operatorname{Im} \Omega_{22} \rightarrow$ $+\infty$. Applying this procedure to the first relation with coefficients $P_{i j k}^{1}$, we obtain the Fourier expansion in coordinate $z_{2}$. The first term of this expansion defines the relation for the genus one theta functions given on an elliptic curve with modulus $\tau=\Omega_{11}$. This relation can be written in terms of the elliptic functions $I_{1}$ and $I_{2}$ as

$$
I_{1}+I_{2}=0
$$




$$
\begin{gathered}
I_{1}=3 \frac{\theta_{00}(0) \theta_{0 \frac{1}{3}}(0) \theta_{0 \frac{1}{3}}^{\prime}(0)}{\theta_{00}\left(z_{1}\right)^{3}}\left(\theta_{00}\left(z_{1}\right) \theta_{0 \frac{2}{3}}\left(z_{1}\right) \theta_{0 \frac{1}{3}}^{\prime \prime}\left(z_{1}\right)-\theta_{00}\left(z_{1}\right) \theta_{0 \frac{1}{3}}\left(z_{1}\right) \theta_{0 \frac{2}{3}}^{\prime \prime}\left(z_{1}\right)-2 \theta_{00}^{\prime}\left(z_{1}\right) \theta_{0 \frac{1}{3}}^{\prime}\left(z_{1}\right) \theta_{0 \frac{2}{3}}\left(z_{1}\right)+\right. \\
\left.+2 \theta_{00}^{\prime}\left(z_{1}\right) \theta_{0 \frac{1}{3}}\left(z_{1}\right) \theta_{0 \frac{2}{3}}^{\prime}\left(z_{1}\right)\right) \\
I_{2}=\frac{1}{\theta_{00}\left(z_{1}\right)^{3}}\left(\theta_{00}\left(z_{1}\right) \theta_{0 \frac{1}{3}}^{\prime}\left(z_{1}\right) \theta_{0 \frac{2}{3}}\left(z_{1}\right)+\theta_{00}\left(z_{1}\right) \theta_{0 \frac{1}{3}}\left(z_{1}\right) \theta_{0 \frac{2}{3}}^{\prime}\left(z_{1}\right)-2 \theta_{00}^{\prime}\left(z_{1}\right) \theta_{0 \frac{1}{3}}\left(z_{1}\right) \theta_{0 \frac{2}{3}}\left(z_{1}\right)\right) \times \\
\times\left(\theta_{00}(0) \theta_{0 \frac{1}{3}}(0) \theta_{0 \frac{1}{3}}^{\prime \prime}(0)-\theta_{0 \frac{1}{3}}(0)^{2} \theta_{00}^{\prime \prime}(0)+2 \theta_{00}(0)\left(\theta_{0 \frac{1}{3}}^{\prime}(0)\right)^{2}\right)
\end{gathered}
$$

where the dependence of the theta functions on modulus $\tau$ is meant. To simplify the relation (5.1) we change the summation variables in theta series (the Koizumi formula [31]), which gives the equation

$$
\begin{gathered}
\sum_{i=0}^{1} \sum_{j=0}^{2} \theta_{\frac{j}{3} 0}(3 z \mid 3 \tau) \theta_{\frac{i}{2}-\frac{j}{3}, 0}^{\prime}(0 \mid 6 \tau) \times \\
\times\left(6 \theta_{00}(0 \mid \tau) \theta_{0 \frac{1}{3}}(0 \mid \tau) \theta_{0 \frac{1}{3}}^{\prime}(0 \mid \tau) \theta_{\frac{i}{2} \frac{2}{3}}^{\prime}(0 \mid 2 \tau)+\theta_{00}(0 \mid \tau) \theta_{0 \frac{1}{3}}(0 \mid \tau) \theta_{0 \frac{1}{3}}^{\prime \prime}(0 \mid \tau) \theta_{\frac{i}{2} \frac{2}{3}}(0 \mid 2 \tau)+\right. \\
\left.+2 \theta_{00}(0 \mid \tau)\left(\theta_{0 \frac{1}{3}}^{\prime}(0 \mid \tau)\right)^{2} \theta_{\frac{i}{2} \frac{2}{3}}(0 \mid 2 \tau)-\theta_{00}^{\prime \prime}(0 \mid \tau)\left(\theta_{0 \frac{1}{3}}(0 \mid \tau)\right)^{2} \theta_{\frac{i}{2} \frac{2}{3}}(0 \mid 2 \tau)\right)=0
\end{gathered}
$$

Therefore, (5.1) is equivalent to the following relation on theta constants:

$$
\begin{gathered}
6 \theta_{00}(0 \mid \tau) \theta_{0 \frac{1}{3}}(0 \mid \tau) \theta_{0 \frac{1}{3}}^{\prime}(0 \mid \tau)\left(\theta_{\frac{2}{3} 0}^{\prime}(0 \mid 6 \tau) \theta_{0 \frac{2}{3}}^{\prime}(0 \mid 2 \tau)+\theta_{\frac{1}{6} 0}^{\prime}(0 \mid 6 \tau) \theta_{\frac{1}{2} \frac{2}{3}}^{\prime}(0 \mid 2 \tau)\right)+ \\
+\left(\theta_{00}(0 \mid \tau) \theta_{0 \frac{1}{3}}(0 \mid \tau) \theta_{0 \frac{1}{3}}^{\prime \prime}(0 \mid \tau)+2 \theta_{00}(0 \mid \tau)\left(\theta_{0 \frac{1}{3}}^{\prime}(0 \mid \tau)\right)^{2}-\theta_{00}^{\prime \prime}(0 \mid \tau)\left(\theta_{0 \frac{1}{3}}(0 \mid \tau)\right)^{2}\right) \times \\
\times\left(\theta_{\frac{2}{3} 0}^{\prime}(0 \mid 6 \tau) \theta_{0 \frac{2}{3}}(0 \mid 2 \tau)+\theta_{\frac{1}{6} 0}^{\prime}(0 \mid 6 \tau) \theta_{\frac{1}{2} \frac{2}{3}}(0 \mid 2 \tau)\right)=0
\end{gathered}
$$

The latter equation can be separated into the other two identities of simpler form:

$$
\begin{gathered}
\theta_{00}(0 \mid \tau) \theta_{0 \frac{1}{3}}(0 \mid \tau) \theta_{0 \frac{1}{3}}^{\prime}(0 \mid \tau)=-2 \imath \sqrt{3} \theta_{\frac{1}{3} 0}(0 \mid 3 \tau)\left(\theta_{\frac{2}{3} 0}^{\prime}(0 \mid 6 \tau) \theta_{0 \frac{2}{3}}(0 \mid 2 \tau)+\theta_{\frac{1}{6} 0}^{\prime}(0 \mid 6 \tau) \theta_{\frac{1}{2} \frac{2}{3}}(0 \mid 2 \tau)\right) \\
\theta_{00}(0 \mid \tau) \theta_{0 \frac{1}{3}}(0 \mid \tau) \theta_{0 \frac{1}{3}}^{\prime \prime}(0 \mid \tau)+2 \theta_{00}(0 \mid \tau)\left(\theta_{0 \frac{1}{3}}^{\prime}(0 \mid \tau)\right)^{2}-\theta_{00}^{\prime \prime}(0 \mid \tau)\left(\theta_{0 \frac{1}{3}}(0 \mid \tau)\right)^{2}= \\
=12 \imath \sqrt{3} \theta_{\frac{1}{3} 0}(0 \mid 3 \tau)\left(\theta_{\frac{2}{3} 0}^{\prime}(0 \mid 6 \tau) \theta_{0 \frac{2}{3}}^{\prime}(0 \mid 2 \tau)+\theta_{\frac{1}{6} 0}^{\prime}(0 \mid 6 \tau) \theta_{\frac{1}{2} \frac{2}{3}}^{\prime}(0 \mid 2 \tau)\right)
\end{gathered}
$$

Analyzing the relation (5.6), we find it to be the consequence of the following identity:

$$
\theta_{\frac{1}{6} \frac{1}{2}}(0 \mid \tau) \theta_{\frac{1}{3} 0}^{\prime}(0 \mid \tau)=\frac{2 \pi}{3} \theta_{\frac{1}{6} \frac{1}{2}}^{2}\left(0 \mid \frac{\tau}{2}\right) \theta_{\frac{1}{6} \frac{1}{2}}^{2}(0 \mid 2 \tau) .
$$

This is a specialization of the Macdonald identity for the case of $B C_{1}$ affine root system [28]. 


\section{Connection with the Ruijsenaars models}

The integrable systems under consideration were proposed in [1, 2] as systems p,q-dual to the elliptic Calogero and Ruijsenaars models. Thus, in some specific trigonometric limits they become dual to the trigonometric Calogero and Ruijsenaars models and the Hamiltonians (2.1) become the Hamiltonians of the rational and trigonometric Ruijsenaars models. The goal of this section is to describe these trigonometric limits and to derive the limiting dependence of the coordinates $\mathbf{z}$ and the elements of the period matrix $\Omega$ on the particle coordinates and momenta $(\mathbf{u}, \mathbf{v})$ of the Ruijsenaars models.

At first, we recall the general construction of the Hamiltonians for the $N$-particle integrable systems p,q-dual to the elliptic Calogero and Ruijsenaars models. The generating function for these Hamiltonians is the genus- $N$ theta function on the Jacobian of the Calogero spectral curve, where $N \times N$ period matrix $T(\mathbf{a})$, depending on $N-1$ flat moduli a, possesses the following property (reflecting the decoupling of the center-of-mass motion):

$$
\sum_{j=1}^{N} T_{i j}(\mathbf{a})=\tau, \quad \forall i,
$$

where $\tau$ does not depend on a. Due to (6.1) the genus- $N$ theta function decomposes into bilinear combinations of genus-one and genus- $g(g=N-1)$ theta functions. Then the claim of [1, 2] is that all the ratios of these genus- $g$ theta functions are Poisson-commuting with respect to the Seiberg-Witten symplectic structure.

In the case of three particles we have $3 \times 3$ period matrix $T$ with the property (6.1). Again, we do not use any Seiberg-Witten data here. Introducing the notations for the genus-2 theta functions with characteristics

$$
\theta^{(2)}[\mathbf{b}] \equiv \theta^{(2)}\left[\begin{array}{c}
\mathbf{b} / 3 \\
\mathbf{0}
\end{array}\right]=\sum_{\mathbf{n} \in \mathbb{Z}^{2}} \mathrm{e}\left(\frac{1}{2}(\mathbf{n}+\mathbf{b} / 3)^{t} \Omega(\mathbf{n}+\mathbf{b} / 3)+(\mathbf{n}+\mathbf{b} / 3) \cdot \mathbf{z}\right), \quad \mathbf{b} \in \mathbb{Z}^{2},
$$

we get the following decomposition of the genus-3 theta function on the Jacobian of the Calogero spectral curve:

$$
\begin{aligned}
\theta^{(3)}(\widetilde{\mathbf{z}} \mid T)=\theta_{00}\left(z_{3} \mid 3 \tau\right)\left(\theta^{(2)}[00]\right. & \left.+\theta^{(2)}[12]+\theta^{(2)}[21]\right)+\theta_{\frac{1}{3} 0}\left(z_{3} \mid 3 \tau\right)\left(\theta^{(2)}[11]+\theta^{(2)}[02]+\theta^{(2)}[20]\right)+ \\
& +\theta_{\frac{2}{3} 0}\left(z_{3} \mid 3 \tau\right)\left(\theta^{(2)}[22]+\theta^{(2)}[01]+\theta^{(2)}[10]\right)
\end{aligned}
$$

where all genus-2 theta functions are functions of $\left(3 z_{1}, 3 z_{2} \mid 9 \Omega\right)$,

$$
z_{1}=\frac{\widetilde{z}_{1}-2 \widetilde{z}_{2}+\widetilde{z}_{3}}{3}, \quad z_{2}=\frac{\widetilde{z}_{1}+\widetilde{z}_{2}-2 \widetilde{z}_{3}}{3}, \quad z_{3}=\frac{\widetilde{z}_{1}+\widetilde{z}_{2}+\widetilde{z}_{3}}{3}
$$

and the genus- 2 period matrix $\Omega$ can be expressed through the diagonal elements $t_{i} \equiv T_{i i}$ as follows:

$$
\Omega=\left(\begin{array}{cc}
t_{2}-\frac{\tau}{3} & \frac{1}{2}\left(t_{1}-t_{2}-t_{3}\right)+\frac{\tau}{6} \\
\frac{1}{2}\left(t_{1}-t_{2}-t_{3}\right)+\frac{\tau}{6} & t_{3}-\frac{\tau}{3}
\end{array}\right)
$$

Then the Poisson-commuting ratios are of the form

$$
\widetilde{H}_{1}=\frac{\theta^{(2)}[11]+\theta^{(2)}[02]+\theta^{(2)}[20]}{\theta^{(2)}[00]+\theta^{(2)}[12]+\theta^{(2)}[21]}, \quad \widetilde{H}_{2}=\frac{\theta^{(2)}[22]+\theta^{(2)}[01]+\theta^{(2)}[10]}{\theta^{(2)}[00]+\theta^{(2)}[12]+\theta^{(2)}[21]} .
$$

The latter expressions are rational functions of the Hamiltonians (2.1) due to the particular case $(g=2, l=3)$ of the following transition formula:

$$
\theta^{(g)}\left[\begin{array}{c}
\mathbf{0} \\
\mathbf{b} / l
\end{array}\right]\left(\mathbf{z} \mid l^{-1} \Omega\right)=\sum_{0 \leq c_{i}<l} \mathrm{e}\left(\frac{\mathbf{b} \cdot \mathbf{c}}{l}\right) \theta^{(g)}\left[\begin{array}{c}
\mathbf{c} / l \\
\mathbf{0}
\end{array}\right](l \mathbf{z} \mid l \Omega), \quad 0 \leq b_{i}<l .
$$


Thus, functions (6.6) can be considered as an equivalent pair of the Hamiltonians.

The described above projection method provides the parameter $\tau$ of an elliptic curve, which can be associated with the elliptic Calogero and Ruijsenaars models. So, it is natural to consider the trigonometric $\operatorname{limit} \operatorname{Im} \tau \rightarrow+\infty$. Additionally, we assume that there exists nonzero positive constant $k>0$ such that

$$
t_{i}-\frac{\tau}{3}=\tau_{i}+k \tau, \quad i=1,2,3
$$

and all $\tau_{i}$ have a finite nonzero limits:

$$
\lim _{\tau \rightarrow+\infty} \tau_{i}=\widetilde{\tau}_{i}
$$

Also, the coordinates $\widetilde{\mathbf{z}}$ on the Jacobian of the Calogero spectral curve are the following functions of particle momenta:

$$
\lim _{\tau \rightarrow+\infty} \widetilde{z}_{i}=v_{i}, \quad i=1,2,3 .
$$

Then for the genus-2 theta function $\theta^{(2)}[\mathbf{b}]$ we have the expansion

$$
\begin{aligned}
\theta^{(2)}\left[b_{1} b_{2}\right]\left(3 z_{1}, 3 z_{2} \mid 9 \Omega\right) & =\sum_{m, n \in \mathbb{Z}} q^{\frac{9}{2} k\left(\left(m+b_{1} / 3\right)^{2}+\left(n+b_{2} / 3\right)^{2}-\left(m+b_{1} / 3\right)\left(n+b_{2} / 3\right)\right)}\left(\frac{q_{1}}{q_{2} q_{3}}\right)^{\frac{9}{2}\left(m+b_{1} / 3\right)\left(n+b_{2} / 3\right)} \times \\
& \times q_{2}^{\frac{9}{2}\left(m+b_{1} / 3\right)^{2}} q_{3}^{\frac{9}{2}\left(n+b_{2} / 3\right)^{2}} \mathrm{e}\left(\left(m+\frac{b_{1}}{3}\right) 3 z_{1}+\left(n+\frac{b_{2}}{3}\right) 3 z_{2}\right),
\end{aligned}
$$

where $q \equiv \mathrm{e}(\tau)$ and $q_{i} \equiv \mathrm{e}\left(\tau_{i}\right)$. This gives the limits of the equivalent Hamiltonians (6.6) in the center of a mass frame:

$$
\begin{gathered}
h_{1}=\lim _{\operatorname{Im} \tau \rightarrow+\infty} q^{-\frac{k}{2}} \widetilde{H}_{1}=\mathrm{e}\left(v_{1}\right) \mathrm{e}\left(\frac{\widetilde{\tau}_{1}}{2}\right)+\mathrm{e}\left(v_{2}\right) \mathrm{e}\left(\frac{\widetilde{\tau}_{2}}{2}\right)+\mathrm{e}\left(v_{3}\right) \mathrm{e}\left(\frac{\widetilde{\tau}_{3}}{2}\right), \\
h_{2}=\lim _{\operatorname{Im} \tau \rightarrow+\infty} q^{-\frac{k}{2}} \widetilde{H}_{2}=\mathrm{e}\left(-v_{1}\right) \mathrm{e}\left(\frac{\widetilde{\tau}_{1}}{2}\right)+\mathrm{e}\left(-v_{2}\right) \mathrm{e}\left(\frac{\widetilde{\tau}_{2}}{2}\right)+\mathrm{e}\left(-v_{3}\right) \mathrm{e}\left(\frac{\widetilde{\tau}_{3}}{2}\right) .
\end{gathered}
$$

In the case of the system dual to the elliptic Calogero model we obtain the following dependence on the particle coordinates $\mathbf{u}$ :

$$
\mathrm{e}\left(\frac{\widetilde{\tau}_{i}}{2}\right)=\prod_{j \neq i} \sqrt{1-\frac{g^{2}}{\left(u_{i}-u_{j}\right)^{2}}},
$$

and functions (6.12), (6.13) are the Hamiltonians of the rational Ruijsenaars model.

In the case of the system dual to the elliptic Ruijsenaars model we obtain

$$
\mathrm{e}\left(\frac{\widetilde{\tau}_{i}}{2}\right)=\prod_{j \neq i} \sqrt{1-\frac{g^{2}}{\sinh ^{2}\left(u_{i}-u_{j}\right)}}
$$

and functions (6.12), (6.13) are the Hamiltonians of the trigonometric Ruijsenaars model.

\section{Lax pair for $N=2$}

In this section we propose $2 \times 2$ Lax pairs for two-particle dual to elliptic models including dual to the Double Elliptic one. In general, using the original Ruijsenaars' idea [29] (see also [34]) one can find the Lax pair for the dual model in the following way. Let an initial model is given by its $N \times N$ Lax matrix $L$ and the problem 
is to find its dual Lax formulation $(\tilde{L})$. Taking into account group-theoretical interpretation of the duality we can formulate it in terms of the linear (eigenvalue) problems

$$
\begin{gathered}
L(p, q) \Psi=\Psi \lambda(U), \quad U=\operatorname{diag}\left(u_{1}, \ldots, u_{N}\right), \\
\tilde{L}(v, u) \Psi^{-1}=\Psi^{-1} f(Q), \quad Q=\operatorname{diag}\left(q_{1}, \ldots, q_{N}\right),
\end{gathered}
$$

where $p, q$ - canonical variables of the initial model, while $v, u$ are those of the dual one and $\Psi$ is the matrix of eigenvectors. From (7.1) it follows that

$$
\operatorname{tr}\left(\lambda^{k}(U) \tilde{L}^{m}\right)=\operatorname{tr}\left(L^{k} f^{m}(Q)\right)
$$

Functions $\lambda$ and $f$ define the free Hamiltonians 1 because $\operatorname{tr} L^{k}$ or $\operatorname{tr} \tilde{L}^{k}$ are symmetric functions of $\lambda\left(u_{i}\right)$ or $f\left(q_{i}\right)$ correspondingly. The free hamiltonians should satisfy the anticanonicity conditions [1].

The idea of our construction is to use the anticanonicity conditions together with (7.1). Let us demonstrate the derivation of the dual Lax matrix in the simplest case $N=2$. In this case $Q=\operatorname{diag}(q,-q), U=\operatorname{diag}(u,-u)$ and there is a single Hamiltonian for each model (and there are no any non-trivial identities discussed above). The Hamiltonians $H(p, q, \nu)$ (its free version is $\left.H_{0}(p)=H(p, q, 0)\right)$ and $\tilde{H}(v, u, \nu)\left(\tilde{H}_{0}(v)\right)$ satisfy the following relations:

$$
H(p, q)=H_{0}(u), \quad \tilde{H}(v, u)=\tilde{H}_{0}(q)
$$

while the anticanonicity condition $\left\{H(p, q), \tilde{H}_{0}(q)\right\}_{p, q}=\left\{H_{0}(u), \tilde{H}(v, u)\right\}_{u, v}$ takes the form:

$$
\partial_{p} H(p, q) \partial_{q} \tilde{H}_{0}(q)=\partial_{u} H_{0}(u) \partial_{v} \tilde{H}(v, u) .
$$

Next, we find $\Psi$ from known $L$. Some freedom in the definition of $\Psi$ can be fixed by condition $\left.\Psi\right|_{\nu=0}=1$. We have

$$
\Psi=\left(\begin{array}{cc}
1 & \frac{\lambda_{2}-L_{22}}{L_{21}} \\
\frac{\lambda_{1}-L_{11}}{L_{12}} & 1
\end{array}\right), \quad \operatorname{det} \Psi=\frac{1}{L_{12} L_{21}}\left(\lambda_{1}\left(L_{22}-\lambda_{2}\right)+\lambda_{2}\left(L_{11}-\lambda_{1}\right)\right),
$$

where $\lambda_{1}=\lambda(u), \lambda_{2}=\lambda(-u)$ and, therefore,

$$
\tilde{L}=\frac{1}{\lambda_{1}\left(L_{22}-\lambda_{2}\right)+\lambda_{2}\left(L_{11}-\lambda_{1}\right)}\left(\begin{array}{cc}
f_{1} L_{12} L_{21}-f_{2}\left(\lambda_{1}-L_{11}\right)\left(\lambda_{2}-L_{22}\right) & L_{12}\left(\lambda_{2}-L_{22}\right)\left(f_{1}-f_{2}\right) \\
-L_{21}\left(\lambda_{1}-L_{11}\right)\left(f_{1}-f_{2}\right) & f_{2} L_{12} L_{21}-f_{1}\left(\lambda_{1}-L_{11}\right)\left(\lambda_{2}-L_{22}\right)
\end{array}\right),
$$

where $f_{1}=f(q), f_{2}=f(-q)$.

Remark Using (7.6) together with (7.2) for $k=m=1$ and (7.4) one can easily reproduce the "trigonometric Calogero - rational Ruijsenaars" models duality [29] for $N=2$ via $\lambda_{1,2}(u)= \pm u$ and $f_{1,2}=\exp ( \pm q \eta)$ :

$$
L=\left(\begin{array}{cc}
p & \frac{\nu^{\prime} \eta}{\sinh (q \eta)} \\
-\frac{\nu^{\prime} \eta}{\sinh (q \eta)} & -p
\end{array}\right) \quad \longrightarrow \quad \tilde{L}=\left(\begin{array}{cc}
e^{\eta v} \sqrt{1-\frac{\nu^{\prime 2} \eta^{2}}{u^{2}}} & -\frac{\nu^{\prime} \eta}{u} \\
\frac{\nu^{\prime} \eta}{u} & e^{-\eta v} \sqrt{1-\frac{\nu^{\prime 2} \eta^{2}}{u^{2}}}
\end{array}\right)
$$

where $\eta$ is the inverse "speed of light".

\footnotetext{
${ }^{1}$ We assume that there exists a coupling constant $\nu$. The free Hamiltonians appear at $\nu=0$. In the examples below we also use notation $\nu^{\prime}=\sqrt{-2} \nu$.
} 


\section{Elliptic Calogero Model and its Dual}

The elliptic two-particle Calogero model is defined by

$$
L=\left(\begin{array}{cc}
p & \frac{\nu^{\prime}}{\operatorname{sn}(q)} \\
-\frac{\nu^{\prime}}{\operatorname{sn}(q)} & -p
\end{array}\right), \quad-\frac{1}{2} \operatorname{det} L=H(p, q)=\frac{1}{2}\left(p^{2}-\frac{\nu^{\prime 2}}{\operatorname{sn}^{2}(q)}\right)=\frac{p^{2}}{2}+\frac{\nu^{2}}{\operatorname{sn}^{2}(q)},
$$

where $\nu^{\prime}=\sqrt{-2} \nu$. With $\lambda(u)=u$ the Hamiltonian $H(p, q)=H_{0}(u)=\frac{1}{2} u^{2}$ and the matrix (7.6) is simplified:

$$
\tilde{L}=\left(\begin{array}{cc}
\frac{1}{2}\left(f_{1}+f_{2}\right)+\frac{L_{11}}{2 u}\left(f_{1}-f_{2}\right) & -\frac{1}{2 u} L_{12}\left(f_{1}-f_{2}\right) \\
-\frac{1}{2 u} L_{21}\left(f_{1}-f_{2}\right) & \frac{1}{2}\left(f_{1}+f_{2}\right)-\frac{L_{11}}{2 u}\left(f_{1}-f_{2}\right)
\end{array}\right),
$$

To determine the function $f$ set $\tilde{H}=\tilde{H}(v, u)=\operatorname{tr} \tilde{L}=\operatorname{cn}(q)$. This gives

$$
f_{1}+f_{2}=\operatorname{cn}(q)
$$

Next, let us use the condition (17.2) $\operatorname{tr}(\lambda(U) \tilde{L})=\operatorname{tr}(L f(Q))$ :

$$
u\left(\tilde{L}_{11}-\tilde{L}_{22}\right)=p\left(f_{1}-f_{2}\right) .
$$

Together with the anticanonicity condition $p \operatorname{cn}^{\prime}(q)=u \frac{\partial \tilde{H}(v, u)}{\partial v}$ it is natural to set

$$
f_{1}-f_{2}=\operatorname{cn}^{\prime}(q)=-\operatorname{sn}(q) \operatorname{dn}(q), \quad \tilde{L}_{11}-\tilde{L}_{22}=\partial_{v} \tilde{H}(v, u)=\dot{u}
$$

Thus,

$$
\lambda(u)=u, \quad f(q)=\frac{1}{2}\left(\operatorname{cn}(q)+\operatorname{cn}^{\prime}(q)\right)=\frac{1}{2}(\operatorname{cn}(q)-\operatorname{sn}(q) \operatorname{dn}(q)) .
$$

Plugging into (7.6) we get the dual Lax matrix

$$
\tilde{L}=\frac{1}{2}\left(\begin{array}{cc}
\operatorname{cn}(q)+\dot{u} & -\operatorname{dn}(q) \frac{\nu^{\prime}}{u} \\
\operatorname{dn}(q) \frac{\nu^{\prime}}{u} & \operatorname{cn}(q)-\dot{u}
\end{array}\right), \quad \dot{u}=\partial_{v} \tilde{H}(v, u) .
$$

Notice that the equation

$$
\operatorname{det} \tilde{L}=f(q) f(-q)
$$

which follows from (7.1) is not an identity since we have already used the anticanonicity condition (it becomes the identity if to express back $\dot{u}$ ). Instead, it provides the equation for the dual Hamiltonian:

$$
\left(\partial_{v} \tilde{H}\right)^{2}=\left(\alpha^{2}(u)-\tilde{H}^{2}\right)\left(\kappa^{\prime 2}+\kappa^{2} \tilde{H}^{2}\right), \quad \alpha^{2}(u)=1-2 \frac{\nu^{2}}{u^{2}},
$$

where $\kappa$ and $\kappa^{\prime}$ are the standard elliptic moduli. Then we get the known answer [1]:

$$
H(v, u)=\alpha(u) \operatorname{cn}\left(v \beta(u) \mid \frac{\kappa \alpha(u)}{\beta(u)}\right), \quad \beta(u)=\sqrt{\kappa^{\prime 2}+\kappa^{2} \alpha^{2}(u)} .
$$

The dual moduli are $\tilde{\kappa}=\frac{\kappa \alpha(u)}{\beta(u)}$ and $\tilde{\kappa}^{\prime}=\frac{\kappa^{\prime}}{\beta(u)}$. It is easy to verify that $\operatorname{dn}(q \mid \kappa)=\beta(u) \operatorname{dn}(\beta(u) v \mid \tilde{\kappa})$. Then the Lax matrix (7.14) takes the form:

$$
\tilde{L}=\frac{1}{2}\left(\begin{array}{cc}
\alpha(\operatorname{cn}(\beta v \mid \tilde{\kappa})-\beta \operatorname{sn}(\beta v \mid \tilde{\kappa}) \operatorname{dn}(\beta v \mid \tilde{\kappa})) & -\beta \operatorname{dn}(\beta v \mid \tilde{\kappa}) \frac{\nu^{\prime}}{u} \\
\beta \operatorname{dn}(\beta v \mid \tilde{\kappa}) \frac{\nu^{\prime}}{u} & \alpha(\operatorname{cn}(\beta v \mid \tilde{\kappa})+\beta \operatorname{sn}(\beta v \mid \tilde{\kappa}) \operatorname{dn}(\beta v \mid \tilde{\kappa}))
\end{array}\right)
$$


Being written in the form (7.14) (in terms of $\dot{u}, u$-variables) the dual Lax matrix is similar to the one of the rational Calogero model. Then it is easy to find its M-operator ( $\operatorname{since} c n(q)$ and $\operatorname{dn}(q)$ are constants on the dual Hamiltonian flow)

$$
\tilde{M}=\left(\begin{array}{cc}
0 & -\operatorname{dn}(q) \frac{\nu^{\prime}}{2 u^{2}} \\
-\operatorname{dn}(q) \frac{\nu^{\prime}}{2 u^{2}} & 0
\end{array}\right)=\left(\begin{array}{cc}
0 & -\beta \operatorname{dn}(\beta v \mid \tilde{\kappa}) \frac{\nu^{\prime}}{2 u^{2}} \\
-\beta \operatorname{dn}(\beta v \mid \tilde{\kappa})) \frac{\nu^{\prime}}{2 u^{2}} & 0
\end{array}\right),
$$

which provides equation of motion from the Lax equation $\dot{\tilde{L}}=[\tilde{L}, \tilde{M}]$ :

$$
\ddot{u}=-\frac{\nu^{\prime 2}}{u^{3}} \operatorname{dn}^{2}(q)=2 \frac{\nu^{2}}{u^{3}} \operatorname{dn}^{2}(q) .
$$

\section{Double Elliptic Model}

In this case the Lax pair can be constructed by a simple generalization of the previously obtained (7.18), (17.19):

$$
\begin{array}{cc}
\tilde{L}=\frac{1}{2}\left(\begin{array}{cc}
\alpha(\operatorname{cn}(\beta v \mid \tilde{\kappa})-\beta \operatorname{sn}(\beta v \mid \tilde{\kappa}) \operatorname{dn}(\beta v \mid \tilde{\kappa})) & -\beta \operatorname{dn}(\beta v \mid \tilde{\kappa}) \varphi(u) \\
\beta \operatorname{dn}(\beta v \mid \tilde{\kappa}) \varphi(u) & \alpha(\operatorname{cn}(\beta v \mid \tilde{\kappa})+\beta \operatorname{sn}(\beta v \mid \tilde{\kappa}) \operatorname{dn}(\beta v \mid \tilde{\kappa}))
\end{array}\right), \\
\tilde{M}=\left(\begin{array}{cc}
0 & \frac{1}{2} \beta \operatorname{dn}(\beta v \mid \tilde{\kappa}) \varphi_{u}^{\prime}(u) \\
\left.\frac{1}{2} \beta \operatorname{dn}(\beta v \mid \tilde{\kappa})\right) \varphi_{u}^{\prime}(u) & 0
\end{array}\right),
\end{array}
$$

where $\alpha=\alpha(u)=\sqrt{1+\frac{\nu^{\prime 2}}{\operatorname{sn}^{2}(u)}}$ while the function $\varphi(u)$ can be chosen to be $\varphi(u)=\frac{\nu^{\prime}}{\operatorname{sn}(u)}$. Another possible choice is $\varphi(u)=\alpha(u)$.

We hope to generalize the suggested approach for $N>2$ in our future papers. The final purpose is to get the Lax pair with spectral parameter. As is well known the elliptic many-body systems are gauge equivalent to the top-like models [32]. The later are described by quadratic algebras [33]. Therefore, another possibility is to find a generalizations of the higher rank Sklyanin algebras and corresponding r-matrices. Some progress towards this idea was made in [7]. One more approach for constructing the Lax pairs $L(z)$ is based on the modification of bundles $\Xi(z)$ via $L(z)=\Xi^{-1}(z) f\left(\partial_{z}\right) \Xi(z)$, where $f$ is some function (which can be elliptic) 35 .

\section{Conclusion}

We found explicit theta-function identities of genus two that stand behind the Poisson commutativity of the Hamiltonians (2.1) of the three-particle integrable systems with elliptic dependence on momenta. The problem of defining the elements of the Poisson structure $P_{i j k}$ was reduced to the problem of solving the system of three partial differential equations with respect to the pair of unknown functions. In addition, we constructed Lax representations for the two-particle systems, which still need to be extended to many-particle case.

An open problem is to find the set of all solutions of the system of three partial differential equations (3.4) and to understand whether the three-particle integrable systems under consideration are unique or not. Solutions of the system (3.4) define the Poisson structure in terms of the coordinates on the Jacobian of the spectral curve and the elements of its period matrix. To establish the connection with the Seiberg-Witten symplectic structure one should transform the Poisson brackets into the canonical ones. This coordinate transformation acts only on the elements of the period matrix and gives an explicit dependence of the period 
matrix on the flat moduli of the corresponding spectral curve. In this way we can get some useful information about the algebraic geometry of the spectral curves. We are going to cover these topics in the subsequent papers.

Acknowledgments. We are grateful to S. Arthamonov, A. Levin and M. Olshanetsky for helpful discussions. The work was partly supported by Ministry of Education and Science of the Russian Federation under contract 8207 (A.Mir., A.Mor., A.Z.), the Brazil National Counsel of Scientific and Technological Development (A.Mor.), by NSh-3349.2012.2 (A.Mir., A.Mor.), by RFBR grants 13-02-00457 (A.Mir.), 1302-00478 (A.Mor.) and 12-01-00482 (G.A., A.Z.), by joint grants 12-02-92108-Yaf (A.Mir., A.Mor.), 13-0291371-ST (A.Mir., A.Mor.), 14-01-93004-Viet (A.Mir., A.Mor.), by leading young scientific groups RFBR 12-01-33071 mol_a_ved (G.A., A.Z.) and by D. Zimin's fund "Dynasty" (A.Z.).

\section{References}

[1] H. W. Braden, A. Marshakov, A. Mironov, and A. Morozov. On Double-Elliptic Integrable Systems 1. A Duality Argument for the case of SU(2). Nuclear Physics B, 573(1):553-572, 2000; hep-th/9906240

[2] A. Mironov and A. Morozov. Commuting Hamiltonians from Seiberg-Witten Theta-Functions. Physics Letters B, 475(1):71-76, 2000; hep-th/9912088

[3] H. W. Braden, A. Marshakov, A. Mironov, and A. Morozov. The Ruijsenaars-Schneider model in the context of Seiberg-Witten theory. Nuclear Physics B, 558(1-2):371-390, 1999; hep-th/9902205

[4] F. Calogero. Exactly solvable one-dimensional many-body problems. Lettere al Nuovo Cimento, 13 (11), 411-416, 1975 .

F. Calogero. On a functional equation connected with integrable many-body problems. Lettere al Nuovo Cimento, 16 (3), 77-80, 1976.

J. Moser. Three integrable Hamiltonian systems connected with isospectral deformations. Advances in mathematics, 16, 1-23, 1975.

M.A. Olshanetsky, A.M. Perelomov. Classical integrable finite-dimensional systems related to Lie algebras. Physics Reports, 71 (5), 313-400, 1981.

[5] S. N. M. Ruijsenaars. Complete integrability of relativistic Calogero-Moser systems and elliptic function identities. Comm. Math. Phys., 110(2):191-213, 1987.

[6] A. Mironov and A. Morozov. Double Elliptic Systems: Problems and Perspectives. 2000; hep-th/0001168

[7] H. W. Braden, A. Gorsky, A. Odesskii, and V. Rubtsov. Double-elliptic dynamical systems from generalized Mukai-Sklyanin algebras. Nuclear Physics B, 633(3):414-442, 2002; hep-th/0111066

V. Fock, A. Gorsky, N. Nekrasov, and V. Rubtsov. Duality in Integrable Systems and Gauge Theories. Journal of High Energy Physics, JHEP07(2000), 2000; hep-th/9906235

[8] N. Seiberg and E. Witten. Electric-magnetic duality, monopole condensation, and confinement in N=2 supersymmetric Yang-Mills theory. Nuclear Physics B, 426(1):19-52, 1994; hep-th/9407087

[9] N. Seiberg and E. Witten. Monopoles, duality and chiral symmetry breaking in N=2 supersymmetric QCD. Nuclear Physics B, 431(3):484-550, 1994; hep-th/9408099

[10] E. Witten. Bound states of strings and p-branes. Nuclear Physics B, 460(2):335-350, 1996; hep-th/9510135

[11] A. Gorsky, A. Marshakov, A. Mironov, and A. Morozov. A Note on Spectral Curve for the Periodic Homogeneous XYZ-Spin Chain. arXiv, 1996; hep-th/9604078

[12] D.-E. Diaconescu. D-branes, monopoles and Nahm equations. Nuclear Physics B, 503(1-2):220-238, 1997; hep-th/9608163 
[13] A. Hanany and E. Witten. Type IIB superstrings, BPS monopoles, and three-dimensional gauge dynamics. Nuclear Physics B, 492(1-2):152-190, 1997; hep-th/9611230

[14] A. Gorsky. Branes and integrability in the N=2 SUSY YM theory. Physics Letters B, 410(1):22-26, 1997; hep-th/9612238

A. Gorsky, S. Gukov, and A. Mironov. Multiscale $\mathrm{N}=2$ SUSY field theories, integrable systems and their stringy/brane origin. Nuclear Physics B, 517(1-3):409-461, 1998; hep-th/9707120

[15] E. Witten. Solutions of four-dimensional field theories via M-theory. Nuclear Physics B, 500(1-3):3-42, 1997; hep-th/9703166

[16] A. Marshakov, M. Martellini, and A. Morozov. Insights and puzzles from branes: 4d SUSY Yang-Mills from 6d models. Physics Letters B, 418(3-4):294-302, 1998; hep-th/9706050

[17] A. Gorsky, S. Gukov, and A. Mironov. SUSY field theories in higher dimensions and integrable spin chains. Nuclear Physics B, 518(3):689-713, 1998; hep-th/9710239

A. Marshakov, and A. Mironov. 5d and $6 \mathrm{~d}$ supersymmetric gauge theories: prepotentials from integrable systems. Nuclear Physics B, 518(1-2):59-91, 1998; hep-th/9711156

[18] Davide Gaiotto. N=2 dualities. Journal of High Energy Physics, 2012(8), 2012; hep-th/0904.2715

[19] A. Gorsky, I. Krichever, A. Marshakov, A. Mironov, and A. Morozov. Integrability and Seiberg-Witten exact solution. Physics Letters B, 355(3-4):466-474, 1995; hep-th/9505035

[20] R. Donagi and E. Witten. Supersymmetric Yang-Mills theory and integrable systems. Nuclear Physics B, 460(2):299-334, 1996; hep-th/9510101

[21] A. Mironov, and A. Morozov. Nekrasov functions and exact Bohr-Sommerfeld integrals. Journal of High Energy Physics, 2010:40, 2010; arXiv:0910.5670 [hep-th]

A. Mironov, and A. Morozov. Nekrasov functions from exact BohrSommerfeld periods: the case of SU(N). Journal of Physics A: Mathematical and Theoretical, 43(19), 2010; arXiv:0911.2396 [hep-th]

[22] Nikita A. Nekrasov and Samson L. Shatashvili. Quantization of integrable systems and four dimensional gauge theories. XVI Congress on Mathematical Physics, pages 265-289, 2009; hep-th/0908.4052

[23] N. Nekrasov, A. Rosly, and S. Shatashvili. Darboux coordinates, Yang-Yang functional, and gauge theory. Nuclear Physics B - Proceedings Supplements, 216(1):69-93, 2011; hep-th/1103.3919

[24] Y. Zenkevich. Nekrasov prepotential with fundamental matter from the quantum spin chain. Physics Letters B, 701(5):630-639, 2011; math-ph/1103.4843

[25] A. Mironov, A. Morozov, Y. Zenkevich, and A. Zotov. Spectral duality in integrable systems from AGT conjecture. JETP Letters, 97(1):45-51, 2013; arXiv:1204.0913 [hep-th]

[26] A. Mironov, A. Morozov, B. Runov, Y. Zenkevich, and A. Zotov. Spectral Duality Between Heisenberg Chain and Gaudin Model. Letters in Mathematical Physics, 103(3):299-329, 2013; arXiv:1206.6349 [hep-th]

[27] A. Mironov, A. Morozov, B. Runov, Y. Zenkevich, and A. Zotov. Spectral dualities in XXZ spin chains and five dimensional gauge theories. to appear.

A. Mironov, A. Morozov, B. Runov, Y. Zenkevich, and A. Zotov. Quantizing spectral dualities, to appear.

[28] I. G. Macdonald. Affine root systems and Dedekind's $\eta$-function. Inventiones mathematicae, 15(2):91-143, 1971.

[29] S. N. M. Ruijsenaars. Action-angle maps and scattering theory for some finite-dimensional integrable systems. Communications in Mathematical Physics, 115(1):127-165, 1988.

[30] H. W. Braden, A. Marshakov, A. Mironov, and A. Morozov. WDVV Equations for 6d Seiberg-Witten Theory and Bi-Elliptic curves. Acta Applicandae Mathematicae, 99(3):223-244, 2007; hep-th/0606035

[31] S. Koizumi. Theta Relations and Projective Normality of Abelian Varieties. American Journal of Mathematics, 98(4):865-889, 1976. 
[32] A.M. Levin, M.A. Olshanetsky and A. Zotov. Hitchin systems - symplectic Hecke correspondence and twodimensional version. Communications in mathematical physics, 236 (1), 93-133, 2003; arXiv:nlin/0110045 [nlin.SI].

A. Levin, M. Olshanetsky, A. Smirnov and A. Zotov. Characteristic classes and Hitchin systems. General construction. Communications in Mathematical Physics, 316 (1), 1-44, 2012; arXiv:1006.0702 [math-ph].

A. Levin, M. Olshanetsky, A. Smirnov and A. Zotov. Calogero-Moser systems for simple Lie groups and characteristic classes of bundles. Journal of Geometry and Physics, 62 (8), 1810-1850, 2012; arXiv:1007.4127 [math-ph].

[33] E.K. Sklyanin. Some algebraic structures connected with the YangBaxter equation. Functional Analysis and its Applications, 16 (4), 263-270, 1982.

A.V. Odesskii and B.L. Feigin. Sklyanin elliptic algebras Functional Analysis and its Applications, 23 (3), 207-214, 1989.

H.W. Braden, V.A. Dolgushev, M.A. Olshanetsky and A.V. Zotov. Classical R-Matrices and the Feigin-Odesskii Algebra via Hamiltonian and Poisson Reductions. Journal of Physics A: Mathematical and General, 36 (25), 6979, 2003; arXiv:hep-th/0301121

Yu. Chernyakov, A. Levin, M. Olshanetsky and A. Zotov. Quadratic algebras related to elliptic curves. Theoretical and Mathematical Physics, 156 (2), 1103-1122, 2008; arXiv:0710.1072 [nlin.SI].

[34] L. Fehér. and C Klimčik. On the duality between the hyperbolic Sutherland and the rational Ruijsenaars-Schneider models. Journal of Physics A: Mathematical and Theoretical, 42 (18), 185202, 2009; arXiv:0901.1983 [math-ph].

L. Fehér. Poisson-Lie interpretation of trigonometric Ruijsenaars duality. Communications in Mathematical Physics, 301 (1), 55-104, 2011; arXiv:0906.4198 [math-ph].

[35] G. Aminov, S. Arthamonov, A. Smirnov and A. Zotov. Modifications of Bundles as Generating Functions of Lax Operators. to appear. 